\title{
Extraction, anti-tyrosinase, and antioxidant activities of the collagen hydrolysate derived from Rhopilema hispidum
}

\begin{abstract}
The study was conducted to determine anti-tyrosinase and antioxidant activities of the extracted collagen hydrolysate $(\mathrm{CH})$ derived from Malaysian jellyfish, Rhopilema hispidum. Collagen was extracted using 1:1 (w:v) $0.1 \mathrm{M} \mathrm{NaOH}$ solution at temperature $25^{\circ} \mathrm{C}$ for $48 \mathrm{hr}$ followed by treatment of 1:2(w:v) distilled water for another $24 \mathrm{hr}$ and freeze-dried. The extracted collagen was hydrolyzed using papain at optimum temperature, $\mathrm{pH}$ and enzyme/substrate ratio [E/S] of $60{ }^{\circ} \mathrm{C}, 7.0$ and 1:50, respectively. $\mathrm{CH}$ was found to exhibit tyrosinase inhibitory activity, DPPH radical scavenging and metal ion-chelating assays up to 64,28 , and $83 \%$, respectively, after $8 \mathrm{hr}$ of hydrolysis process. The molecular weight of $\mathrm{CH}$ was found $<10 \mathrm{kDa}$ consisting of mainly Gly (19.219\%), Glu (10.428\%), and Arg (8.848\%). The UV-visible spectrum analysis showed a major and minor peak at 218 and $276 \mathrm{~nm}$, accordingly. The FTIR spectroscopy confirmed the amide groups in $\mathrm{CH}$. The SEM images demonstrated spongy and porous structure of $\mathrm{CH}$. In the cytotoxicity study, $\mathrm{CH}$ has no cytotoxicity against mouse embryonic 3T3 fibroblast cell line with IC50 value $>500 \mu \mathrm{g} / \mathrm{ml}$. Results revealed that the $\mathrm{CH}$ generated from this study has a potential to be developed as active ingredient in cosmeceutical application.
\end{abstract}

Keyword: Antioxidant; Anti-tyrosinase; Collagen hydrolysate $(\mathrm{CH})$; DPPH 